\title{
Statin Therapy and the Expression of Genes that Regulate Calcium Homeostasis and Membrane Repair in Skeletal Muscle
}

\author{
Annette Draeger, ${ }^{*}$ Verónica Sanchez-Freire, ${ }^{*}$ \\ Katia Monastyrskaya, ${ }^{*}$ Hans Hoppeler, ${ }^{*}$ \\ Matthias Mueller, ${ }^{*}$ Fabio Breil, ${ }^{*}$ \\ Markus G. Mohaupt, ${ }^{\dagger}$ and Eduard B. Babiychuk* \\ From the Institute of Anatomy, and the Department of \\ Nephrology/Hypertension, ${ }^{\dagger}$ Inselspital, University of Bern, \\ Bern, Switzerland
}

In skeletal muscle of patients with clinically diagnosed statin-associated myopathy, discrete signs of structural damage predominantly localize to the $T$ tubular region and are suggestive of a calcium leak. The impact of statins on skeletal muscle of non-myopathic patients is not known. We analyzed the expression of selected genes implicated in the molecular regulation of calcium and membrane repair, in lipid homeostasis, myocyte remodeling and mitochondrial function. Microscopic and gene expression analyses were performed using validated TaqMan custom arrays on skeletal muscle biopsies of 72 age-matched subjects who were receiving statin therapy $(n=38)$, who had discontinued therapy due to statin-associated myopathy $(n=14)$, and who had never undergone statin treatment $(n=20)$. In skeletal muscle, obtained from statin-treated, non-myopathic patients, statins caused extensive changes in the expression of genes of the calcium regulatory and the membrane repair machinery, whereas the expression of genes responsible for mitochondrial function or myocyte remodeling was unaffected. Discontinuation of treatment due to myopathic symptoms led to a normalization of gene expression levels, the genes encoding the ryanodine receptor 3 , calpain 3 , and dystrophin being the most notable exceptions. Hence, even in clinically asymptomatic (non-myopathic) patients, statin therapy leads to an upregulation in the expression of genes that are concerned with skeletal muscle regulation and membrane repair. (Am J Pathol 2010, 177:291-299; DOI: 10.2353/ajpath.2010.091140)
Statins are generally regarded as the treatment of choice for hypercholesterolaemia. Consequently, it is not surprising that the annual prescription of these drugs has exceeded the 100-million-mark during the past two decades. ${ }^{1,2}$ Notwithstanding their excellent safety profile, adverse effects on skeletal muscle are by no means infrequent. Since statin-associated myopathy is not consistently coupled with an elevation in the serum levels of creatine kinase, the muscular disturbances often remain undiagnosed. ${ }^{3-5}$ Although minor in occurrence, their manifestation has a negative bearing on physical activity and patient compliance with this life-saving therapy. ${ }^{6}$ Recent data demonstrate a genetic susceptibility for statin associated myopathy, ${ }^{7}$ which can be linked to a single nucleotide polymorphism of the SCLO1B1 gene. Carriers of the SCLO1B1*5 allele are at a twofold relative risk of developing statin-associated myopathy. ${ }^{8}$ The effects of statins on gene expression in statin-naïve skeletal muscle have previously been investigated in cell culture ${ }^{9-11}$ and the effects of statin treatment in combination with eccentric exercise studied on skeletal muscle of eight healthy volunteers. ${ }^{12}$

Observational studies have revealed $\sim 10 \%$ of patients to develop statin-associated myopathy. ${ }^{4}$ But it is not yet known what distinguishes the muscle of these individuals from that of the $\sim 90 \%$ who remain asymptomatic.

In a previous study of ours, we demonstrated vacuolization of the T-tubular system to be a recurrent feature and to be more prevalent among patients who had been

Supported by the Swiss National Science Foundation (SNSF): National Research Programme NRP 53 "Musculoskeletal Health-Chronic Pain" to A.D. (grant 405340-104679/1) and SNSF grants to K.M. (320000-111778), to E.B. (3100A0-121980/1), and A.D. (320030_128064/1).

Accepted for publication March 22, 2010.

A.D. has received consultancy fees from PPD (Jan-Apr 2008) for participation in a phase I study, unrelated to the present submission. None of the other authors declare any relevant financial relationships.

Supplemental material for this article can be found on http://ajp. amjpathol.org

Address reprint requests to Prof. Annette Draeger, Institute of Anatomy, University of Bern, Baltzerstr. 2, 3012 Bern, Switzerland. E-mail: draeger@ ana.unibe.ch. 
clinically diagnosed as having statin-associated myopathy. ${ }^{13}$ The biopsies of these patients were characterized by a significant upregulation of the ryanodine receptor 3 gene, which is suggestive of an intracellular calcium leak. ${ }^{13}$ These findings prompted us to investigate the impact of statins on the expression of selected genes involved in the molecular regulation of calcium and membrane repair.

Other gene families, which are potentially affected by cholesterol-lowering treatment are those involved in the regulation of lipid homeostasis and/or mitochondrial function. ${ }^{14-16}$ In addition, the expression of genes involved in myocyte remodeling was assessed to determine the extent of cellular reorganization.

\section{Materials and Methods}

\section{Patients}

This study was conducted with the approval of the Ethics Committee of the Canton of Bern, Switzerland. All subjects gave informed consent. Samples of the vastus lateralis muscle were collected from a total of 72 individuals. They belonged to three different groups: Group 1: 20 subjects who had never undergone statin therapy, (mean age $64 \pm 13$ years; $20 \%$ female). 8 of these subjects were partially analyzed in our previous study ${ }^{13}$ and 12 subjects took part in a longitudinal study on the effects of eccentric training in the elderly. ${ }^{17}$ Their baseline biopsy was used in the present study. Group 2: 14 patients (mean age $60 \pm 11$ years, $50 \%$ female) who had a history of clinically diagnosed statin-associated myopathy, and who had voluntarily discontinued their statin treatment for a minimum of 3 weeks (median 12 weeks, range 3 to 300 weeks) before the time of their biopsy. Eleven biopsies in this group were partially analyzed in our previous study, ${ }^{13} 3$ biopsies were new. Group 3: 38 statin-treated patients (mean age $61 \pm 11$ years, 37\% female), 14 of whom had a clinically diagnosed history of statin-associated myopathy (2 new) and 19 patients who received statin therapy without muscle complaints (6 new).

Subjects were identified as having statin-associated myopathy by clinical criteria, consistent with the Recommendations of the Muscle Safety Expert Panel. ${ }^{18}$ Patients suffering from statin-associated myopathy were referred to the Department of Nephrology and Hypertension for the evaluation of alternative treatment options. Asymptomatic statin-treated subjects were recruited via newspaper advertisements. The vastus lateralis muscle was biopsied at mid-thigh level. ${ }^{19}$ In brief, the lateral portion of one thigh was anesthetized (1\% xylocaine) and a small incision made through the skin and underlying fascia and $\sim 50$ to $100 \mathrm{mg}$ of tissue biopsied using a 14 gauge needle. The muscle sample was immediately frozen in liquid nitrogen and/or chemically fixed for microscopy.

Since cross-sectional biopsies were used throughout the study, none of the biopsies compares the pre- with the post-treatment condition of any individual patient.

\section{Processing of Tissue}

An average of 3 tissue blocks $(\sim 3 \times 6 \mathrm{~mm})$ per patient were fixed and processed for semithin sectioning as well as ultrastructural analysis in the electron microscope. ${ }^{13}$ Whenever sufficient material was available, an additional 1 to 2 blocks were processed for ultracryomicrotomy and immunohistochemistry. ${ }^{20}$

The polyclonal antibodies against annexin A6 have been described previously. ${ }^{21}$ Monoclonal antibodies against annexin $A 6$ and the dihydropyridine receptor were obtained from Invitrogen (Basel, Switzerland), a polyclonal antibody against dysferlin was from Imgenex (Luceme, Switzerland). Secondary antibodies were purchased from Jackson Laboratories (Milan, La Roche, Switzerland) (Cy3-coupled) and Molecular Probes (Invitrogen; Alexa-coupled). Nuclear labeling was performed using the Hoechst 33342 (Sigma, Buchs, Switzerland) stain.

\section{Analysis of Tissue}

The specimen were evaluated by an observer who was skilled in light and electron microscopy but blinded as to the patient groups. On transverse semithin sections, between 200 and 400 individual fibers were analyzed per biopsy. The number of muscle fibers displaying structural abnormalities was expressed as a percentage of the total number of fibers per section in the light microscope. The threshold for significant muscle injury was arbitrarily determined to be $\geq 2 \%$ damaged fibers/biopsy. ${ }^{13}$

\section{RNA Isolation and Gene Expression Studies}

Total RNA was isolated from the muscle biopsies using RNeasy Fibrous Tissue Kit (Qiagen, Hombrechtikon, Switzerland) according to the manufacturer's instructions treating the samples with DNase I (Qiagen) to avoid any genomic DNA contamination. Total RNA concentration was measured using spectrophotometry at $260 \mathrm{~nm}$ (DU 530, Beckman Coulter, Nyon, Switzerland), the purity was determined with the $260_{A} / 280_{A}$ range and controlled by agarose gel electrophoresis. CDNA was synthesized using random hexamer primers and High Capacity cDNA Reverse Transcription Kit (Applied Biosystems, Rotkreuz, Switzerland).

Expression studies were performed using validated TaqMan custom Arrays in a 384-well micro fluidic card format allowing simultaneous analysis of 47 human genes and the reference gene 18S rRNA in two replicates. Eight cDNA samples using $100 \mathrm{ng}$ of starting total RNA were analyzed using each card. Forty-one genes were selected for analysis (Table 1).

Reactions were run on the Applied Biosystems $7900 \mathrm{HT}$ Fast Real-Time PCR System, at the default setting program $\left(50^{\circ} \mathrm{C} 2\right.$ minutes, $94.5^{\circ} \mathrm{C} 10$ minutes; $97^{\circ} \mathrm{C}, 30 \mathrm{~s}$; $59.7^{\circ} \mathrm{C}, 60 \mathrm{~s} ; 40$ cycles). Data acquisition was performed according to the manufacturer's suggestions. Expression values for target genes were normalized to the concentration of $18 \mathrm{~S}$ rRNA. Gene expression values were calcu- 
Table 1. Genes Selected for Molecular Analysis

\begin{tabular}{|c|c|c|c|}
\hline \multirow[b]{2}{*}{ Genes } & \multirow[b]{2}{*}{ Unique identifiers } & \multicolumn{2}{|c|}{$P$ values } \\
\hline & & Figure 2 & Figure 4 \\
\hline \multicolumn{4}{|l|}{ Ion channels, exchangers, transporters, pumps } \\
\hline Inositol-1,4,5-triphosphate receptor 1 & ITPR1 Hs00181881_m1 & 0.000 & 0.051 \\
\hline Inositol-1,4,5-triphosphate receptor 2 & ITPR2 Hs00181916_m1 & 0.000 & 0.306 \\
\hline Inositol-1,4,5-triphosphate receptor 3 & ITPR3 Hs00609908_m1 & 0.000 & 0.051 \\
\hline Ryanodine receptor 1 & RYR1 Hs00166991_m1 & 0.079 & 0.180 \\
\hline Ryanodine receptor 3 & RYR3 Hs00168821_m1 & 0.001 & 0.017 \\
\hline L-type Ca-channel alpha1 S subunit = dihydropyridine receptor & CACNA1S Hs00163̄885_m1 & 0.001 & 0.457 \\
\hline $\mathrm{Na}+/ \mathrm{K}^{+}$ATPase alpha1 isoform & ATP1A1 Hs00167556_m1 & 0.062 & 0.781 \\
\hline $\mathrm{Na} / \mathrm{Ca}$ exchanger ATPase & ATP2B1 Hs01001488_m1 & 0.004 & 0.120 \\
\hline Sarco-endoplasmic reticulum transporting Ca2+ ATPase 1 & ATP2A1 Hs00188877_m1 & 0.095 & 0.368 \\
\hline Sarco-endoplasmic reticulum transporting Ca2+ ATPase 2 & ATP2A2 Hs00544877_m1 & 0.003 & 0.985 \\
\hline Sarco-endoplasmic reticulum transporting Ca2+ ATPase 3 & ATP2A3 Hs00193090_m1 & 0.001 & 0.043 \\
\hline \multicolumn{4}{|l|}{$\mathrm{Ca}^{2+}$-regulated proteins, annexin family and binding partners } \\
\hline Annexin 1 & ANXA1 Hs00167549_m1 & 0.003 & 0.169 \\
\hline Annexin 2 & ANXA2 Hs00743063-s1 & 0.000 & 0.174 \\
\hline Annexin 3 & ANXA3 Hs00192983_m1 & 0.140 & 0.396 \\
\hline Annexin 4 & ANXA4 Hs00154040_m1 & 0.001 & 0.377 \\
\hline Annexin 5 & ANXA5 Hs00154054_m1 & 0.007 & 0.169 \\
\hline Annexin 6 & ANXA6 Hs00241762_m1 & 0.000 & 0.592 \\
\hline Annexin 7 & ANXA7 Hs00559413_m1 & 0.684 & 0.334 \\
\hline S100A6 (calcyclin) & S100A6 Hs00170953_m1 & 0.065 & 0.849 \\
\hline S100A10 & S100A10 Hs00237010_m1 & 0.003 & 0.623 \\
\hline S100A11 & S100A11 Hs00271612_m1 & 0.583 & 0.478 \\
\hline \multicolumn{4}{|l|}{ Lipid metabolism } \\
\hline Hydroxy-methyl-glutaryl-CoA reductase & HMGCR Hs00168352_m1 & 0.000 & 0.396 \\
\hline Peroxisome-proliferator-activated receptor alpha & PPARA Hs00231882_m1 & 0.065 & 0.162 \\
\hline LDL receptor & LDLR Hs00181192_m1 & 0.004 & 0.717 \\
\hline Sterol regulatory element binding transcription factor 1 & SREBF1 Hs01088691_m1 & 0.070 & 0.163 \\
\hline SREBP cleavage-activating protein & SCAP Hs00378725_m1 & 0.000 & 0.152 \\
\hline Sterol O-acyltransferase 1 & SOAT1 Hs0016207̄̄_m1 & 0.120 & 0.323 \\
\hline \multicolumn{4}{|l|}{ Sarcolemma-associated } \\
\hline Caveolin 3 & CAV3 Hs00154292_m1 & 0.725 & 0.592 \\
\hline Dysferlin & DYSF Hs00243339_m1 & 0.000 & 0.986 \\
\hline Dystrophin & DMD Hs00187805_m1 & 0.000 & 0.003 \\
\hline \multicolumn{4}{|l|}{ Calpains } \\
\hline Calpain1 & CAPN1 Hs00559804_m1 & 0.003 & 0.362 \\
\hline Calpain2 & CAPN2 Hs00156251_m1 & 0.372 & 0.247 \\
\hline Calpain3 & CAPN3 Hs00544982_m1 & 0.000 & 0.002 \\
\hline \multicolumn{4}{|l|}{ Mitochondrial metabolism } \\
\hline Cytochrome c oxidase I, mitochondrially encoded & MT-CO1 Hs02596864-g1 & 0.681 & 0.699 \\
\hline $\mathrm{NADH}$ gene $1, \mathrm{mt}$ encoded & MT-ND1 Hs02596873-s1 & 0.755 & 0.005 \\
\hline ATP synthase $6, \mathrm{mt}$ encoded & MT-ATP6 Hs150324 & 0.494 & 0.030 \\
\hline \multicolumn{4}{|l|}{ Remodeling, repair } \\
\hline Myosin-heavy chain, embryonic & MYH3 Hs00159463_m1 & 0.649 & 0.351 \\
\hline Collagen 1 alpha1 & COL1A1 Hs0107674̄6_m1 & 0.335 & 0.938 \\
\hline Basic fibroblast growth factor & FGFR4 Hs00242558_m̄1 & 0.052 & 0.211 \\
\hline Atrogin-1 & FBXO32Hs01041408_m1 & 0.063 & 0.938 \\
\hline \multicolumn{4}{|l|}{ Structural } \\
\hline Alpha skeletal actin & ACTA1 Hs00559403_m1 & 0.022 & 0.115 \\
\hline
\end{tabular}

lated based on the comparative threshold cycle (Ct) method. In short, the Ct data for the selected genes and $18 \mathrm{~S}$ rRNA in each sample were used to create $\Delta \mathrm{Ct}$ values $\left(\mathrm{Ct}_{\text {gene }}-\mathrm{Ct}_{18 \mathrm{~S} \text { rRNA }}\right)$. Thereafter, $\Delta \Delta \mathrm{Ct}$ values were calculated by subtracting the $\Delta \mathrm{Ct}$ of the calibrator from the $\mathrm{Ct}$ value of each target.

\section{Statistics}

Numerical data are expressed as mean values together with the SEM For mRNA expression analysis, the Normality was studied with the Kolmogorov-Smirnov and Shapiro-Wilk tests ( $\alpha$ set to 0.05), with the Lilliefors Significance Correction. Since the genes do not display a normal distribution, the Mann Whitney U-test was performed. The statistical analyses were performed using SPSS version 15 for Windows (Systat Software Inc, San Jose, CA). The level of significance was set at $P<0.05$. Statistical values were displayed using the GraphPad Prism software version 5.02.

A covariate regression model for the effect of gender and the statin- and myopathy-related condition, respectively, was applied.

\section{Results}

The aim of the present study was to assess the impact of statin therapy on skeletal muscle obtained from non- 
A

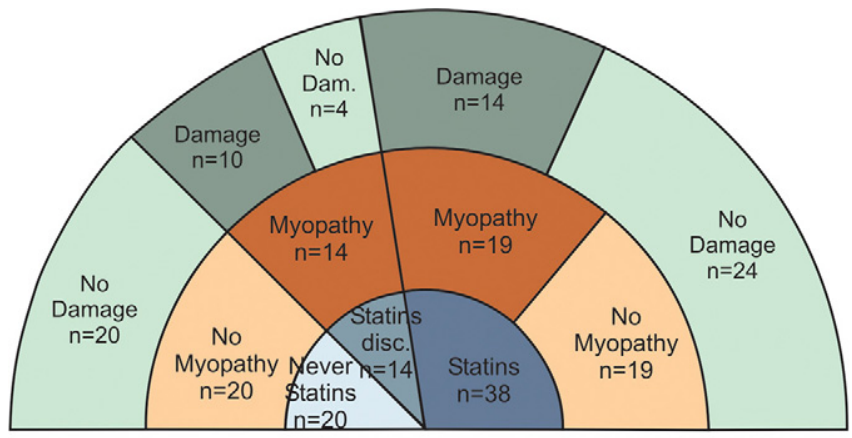

Never statins

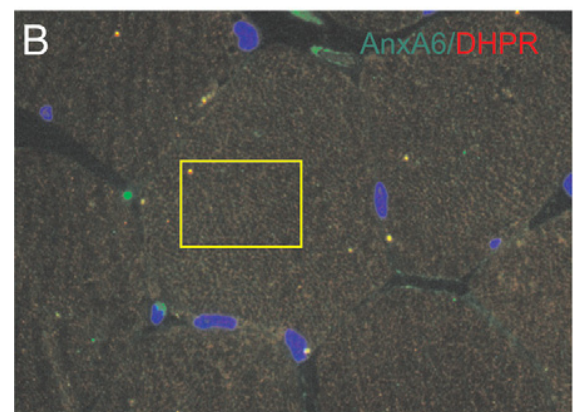

Statin therapy: myopathy \& structural damage
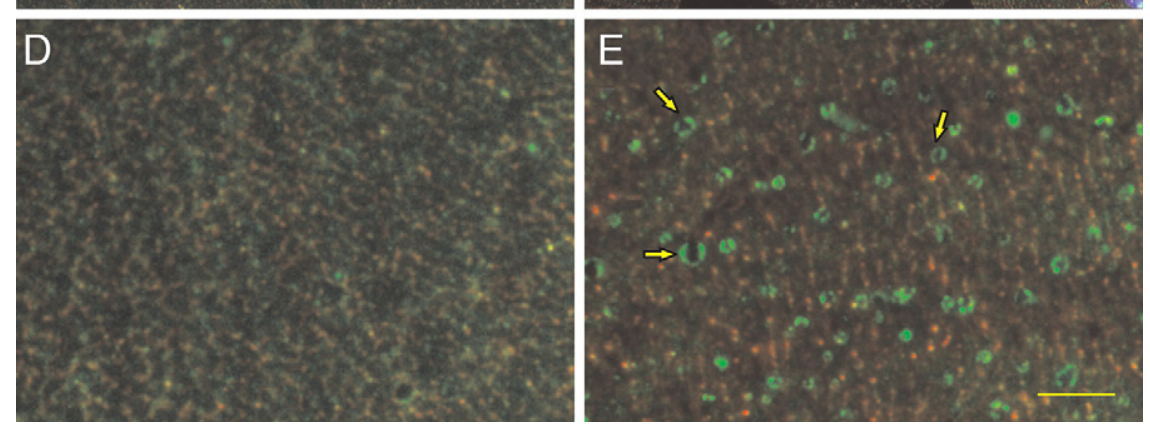

Figure 1. Statin therapy, myopathy and structural damage. A: Color-coded display of the correlation between a clinical diagnosis of myopathy (red) vs no myopathy (pink) and the histopathological manifestation of skeletal muscle damage (dark green) vs no damage (pale green) in the three groups of patients: ongoing statin therapy (dark blue), discontinued statin therapy (turquoise), and no history of statin therapy (pale blue). B-E: Fluorescence micrographs of transverse ultrathin cryosections of skeletal muscle double-labeled with antibodies against T-tubular markers [rabbit anti-annexin A6 (green)] and mouse anti-dihydropyridine receptor (DHPR:red). The nuclei are labeled with Hoechst stain (blue). $\mathbf{B}$ and $\mathbf{D}$ represent tissue that was derived from an individual who had never undergone statin therapy (control). $\mathbf{C}$ and $\mathbf{E}$ represent structurally damaged tissue that was derived from a patient who was undergoing statin therapy and who was suffering from myopathy. The boxed areas in $\mathbf{B}$ and $\mathbf{C}$ are enlarged in $\mathbf{D}$ and E. The tissue of the control subject has a normal appearance, whereas that of the statin-treated patient shows T-tubular vacuolization (arrows). Scale bars: $50 \mu \mathrm{m}(\mathrm{A}, \mathrm{B}), 10 \mu \mathrm{m}(\mathrm{C}, \mathrm{D})$.

myopathic statin-treated patients. Consequently, the skeletal muscle samples of all individuals $(n=72)$ were screened in the light microscope for signs of structural injury, arbitrarily defined as significant if more than $\geq 2 \%$ fibers per biopsy were damaged. ${ }^{13}$ The findings of this analysis correlated well with the clinical data (Figure 1A). If the size of the biopsy was sufficiently large, then a portion of the material was cryosectioned for a highresolution immunohistochemical analysis of markers of the T-tubular system ${ }^{21,22}$ (Figure 1, B-E). Skeletal muscle from 14 statin-treated patients suffering from myopathy (Figure 1A, dark green), as well as from 10 subjects who had discontinued statin therapy (Figure 1A, dark green), displayed vacuolizations within the T-tubular system (Figure 1, B-E). As described previously, ${ }^{13}$ these injuries were modest, typically confined to the intracellular space and did not extend to the sarcolemma. Age-matched controls who had never been treated with statins did not suffer from myopathy and showed no muscular damage.

The 41 genes that were selected for molecular analysis (Table 1), encode proteins that are implicated in the regulation of $\left[\mathrm{Ca}^{2+}\right]_{\text {i }}$, in T-tubular and sarcolemmal structure and membrane repair, in the regulation of lipid metabolism, in myocyte remodeling and in mitochondrial activity.
Initially, we compared skeletal muscle samples of asymptomatic patients without structural damage ( $n=19$, Figure 2A, red) with samples from subjects who had never undergone statin therapy ( $n=20$, Figure 2A pink). Despite the absence of T-tubular vacuolation, many of the genes that are implicated in calcium homeostasis were significantly up-regulated in statin-treated individuals. They include genes for the regulation of $\mathrm{Ca}^{2+}$ influx: inositol-3 receptors (ITPR1\&2\&3), ryanodine receptor 3 (RYR3), the dihydropyridine receptor (CACNA1S), for $\mathrm{Ca}^{2+}$ uptake: sarco-endoplasmic ATPases (ATP2A2\&3) or for $\mathrm{Ca}^{2+}$ efflux: the $\mathrm{Na} / \mathrm{Ca}$ exchanger ATPase (ATP2B1) (Figure 2B). Not affected were the ryanodine receptor 1 (RYR1), the $\mathrm{Na} / \mathrm{K}$-exchanger (ATP1A1) and the sarco-endoplasmic ATPase 1 (ATP2A1). Consistent with these findings, the geneexpression levels of the principal $\mathrm{Ca}^{2+}$-dependent proteases of skeletal muscle, calpains $1 \& 3$, (CAPN1, 3), were likewise raised, whereas those of calpain 2 (CAPN2) remained unchanged (Figure 2B).

Selective marker proteins, such as dystrophin and caveolin 3 are exclusively expressed at the lateral sarcolemma and distinguish the load-bearing part of the plasma membrane from the current-conducting T-tubular membrane system, to which the annexin protein family is 
A

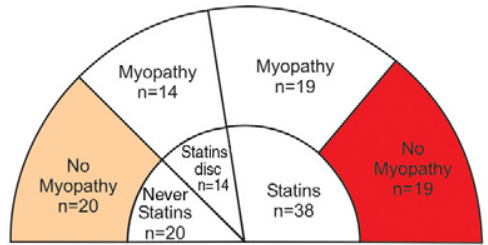

B

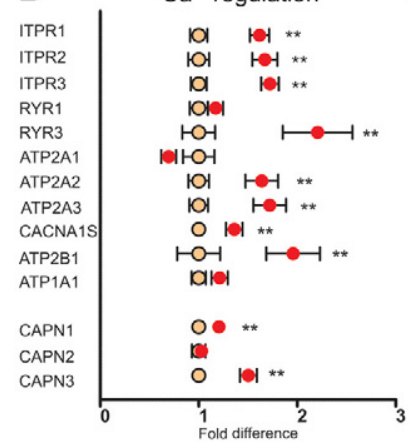

D

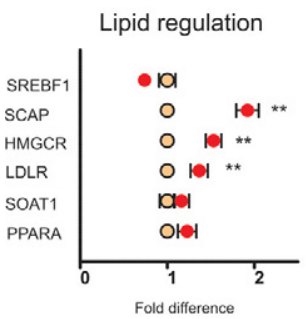

C T-tubular \& sarcolemmal structure

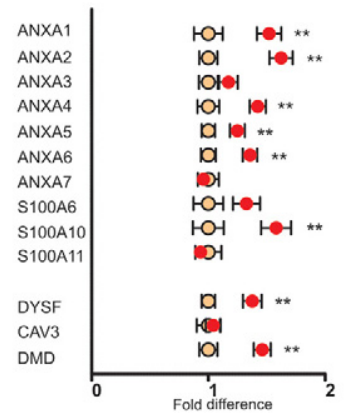

$\mathrm{E}$

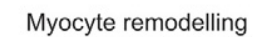

\& mitochondrial activity

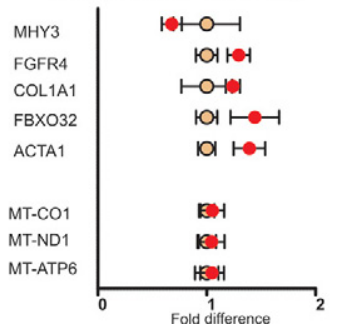

Figure 2. Changes in the gene expression (mRNA) levels of key marker proteins within the skeletal muscle biopsies of non-myopathic patients undergoing statin therapy. A: Color-coded display of the two categories of patients whose biopsies were analyzed for the mRNA levels of genes encoding proteins indicated in B-E. These patients included the 20 controls who had never been treated with statins (pink) and the 19 asymptomatic ones who were receiving statin therapy (red). B-E: The mRNA expression levels of 41 genes were determined using a custom Taqman microarray system. The symbols (color-coded as in a) depict the Fold Differences [reference group: subjects who had never received statin therapy (pink)] for each gene product, normalized to the level of $18 \mathrm{~S}$ RNA (mean values \pm SEM). B: Most of the genes implicated in $\mathrm{Ca}^{2+}$ homeostasis are up-regulated (ITPR1\&2\&3, RYR1\&3, ATPA2\&3, CACNA1, ATP2B1). Several proteases (calpains 1\&3; CAPN1\&3) are up-regulated. C: Genes encoding structural proteins of the T-tubular system: members of the annexin family (ANXA1\&2\&4\&6, S100A6, S100A10), for dysferlin (DYSF) and for dystrophin (DMD), are highly significantly up-regulated. D: Cholesterol-sensors SREBP cleavage-activating protein (SCAP) and the HMG COA reductase (HMGCR), as well as the LDL receptor (LDLR) are highly significantly upregulated. E: Genes that are often associated with the remodeling of muscle fibers (FGFR4, COL1A1, and FBXO32), or with mitochondrial activity (MT-CO1 and MT-ND1 and MT-ATP6), or actin (ACATA1) are not significantly up-regulated. For identification of gene products and $P$ values see Table 1.

bound in a $\mathrm{Ca}^{2+}$-dependent manner. ${ }^{21}$ The membranes of the T-tubular system are particularly susceptible to cholesterol depletion. ${ }^{22}$ Genes encoding members of the annexin protein family (ANXA1-7) and their binding partners (S100A6\&10\&11) as well as dysferlin (DYSF) were up-regulated, whereas the expression levels of caveolin 3 (CAV3) remained unchanged (Figure 2C). Although transmission electron microscopy previously showed the sarcolemma to be intact, ${ }^{13}$ the gene encoding dystrophin (DMD) was significantly up-regulated (Figure 2C).

Since in 19 patients the cholesterol synthesis was pharmaceutically lowered, genes encoding cholesterol-sensing proteins [sterol regulatory-element-binding-protein cleav- age-activating protein (SCAP) and hydroxy-methyl-glutarylCoA reductase (HMGCR), were, not surprisingly, markedly up-regulated; so too, was the gene for the LDL receptor (LDLR), which mediates the cellular uptake of cholesterol from the serum (Figure 2D). Genes encoding either a transcription factor associated with cholesterol synthesis: sterol regulatory element binding transcription factor 1 (SREBF1), or a protein involved in the esterification of cholesterol (SOAT1) were not up-regulated, neither was the peroxisome-proliferator-activated receptor $\alpha$ (PPARA), which encodes enzymes that are involved in the intracellular oxidation of fatty acids, including those of the cytochrome p450 pathways (Figure 2D).

Genes encoding proteins that are involved in the remodeling of either injured skeletal muscle myocytes, such as an embryonic myosin isoform [myosin heavychain 3, (MHY3)] which is synthesized de novo, or extracellular matrix proteins [fibroblast-growth -factor-receptor 4 (FGFR4)] and collagen type 1 (COL1A1) were not up-regulated; neither was the gene encoding the molecular mediator of muscular atrophy, atrogin 1 (FBXO32). Likewise unaffected were the gene expression levels of 3 proteins that influence mitochondrial activity: cytochrome c oxidase (MT-CO1), NADH-ubiquinone oxidoreductase (MT-ND1) and ATP synthase 6 (MT-ATP6). The gene encoding $\alpha$-skeletal muscle actin (ACTA1) was slightly upregulated ( $P=0.022$; Figure 2E).

In a covariance analysis (Supplemental Table S1 at http://ajp.amjpathol.org) gene expression changes did not appear to be linked to gender. However, some differences in values for the female gender (Group: No Myopathy Never Statin versus No Myopathy Statins) were noted and attributed to the low number of female subjects in that particular group.

The upregulation of genes encoding proteins that are involved in $\mathrm{Ca}^{2+}$-homeostasis, in combination with a dilated T-tubular system, might lead to an activation of membrane repair processes, as is implied by the upregulation of "membrane repair" genes dysferlin and dystrophin. ${ }^{23}$ Therefore, ultrathin cryosections of biopsies from untreated subjects (Figure 3, A-F) and from statintreated patients (Figure 3, G-L) were analyzed immunohistochemically. Immunostaining for dysferlin showed the sarcolemma to be uninterrupted in untreated and statintreated patients alike (Figure 3, B, C, H, I). However, in the statin-treated patients, dysferlin was also detected at intracellular sites that did not coincide with the annexin A6-demarcated T-tubular system (Figure 3, H and I). We observed that a redistribution of dysferlin was more obvious in patients whose T-tubular system displayed signs of vacuolization.

In patients who had chosen to discontinue their course of treatment with statins (Figure $4 \mathrm{~A}, n=14$, turquoise), an overall normalization of the gene expression profiles was observed. Notable exceptions relating to $\mathrm{Ca}^{2+}$ homeostasis (Figure 4B) were the genes encoding the ryanodine 3 receptor (RYR3), the SERCA 3 pump (ATP2A3) and calpain 3 (CAPN3), all of which were up-regulated. With the exception of dystrophin (DMD), the expression levels of genes encoding proteins of the sarcolemma and the T-tubular system 

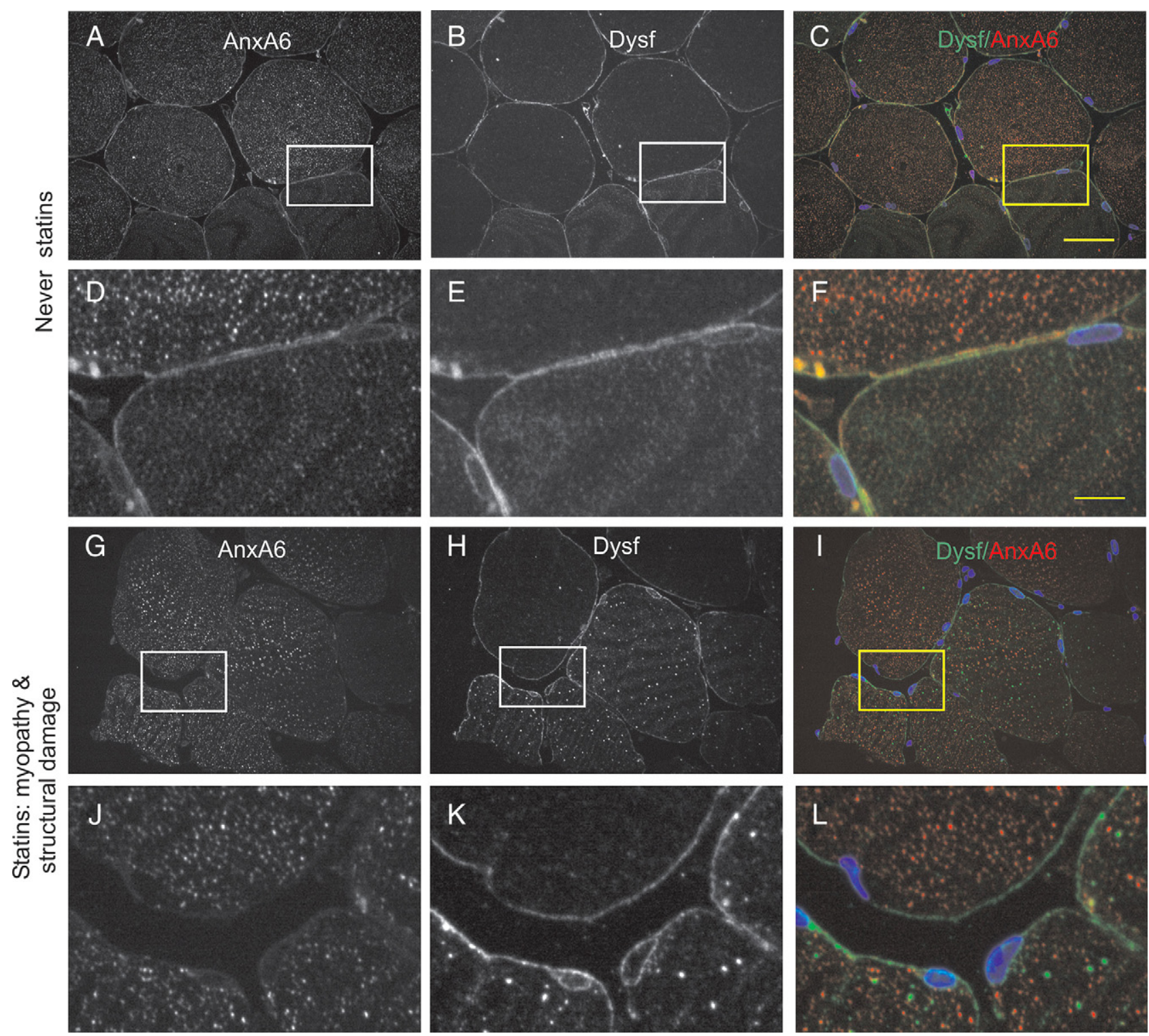

Figure 3. Intracellular accumulation of dysferlin in a biopsy of a myopathic patient undergoing statin therapy. A-L: Fluorescent micrographs of transverse ultrathin cryosections, double-labeled with a monoclonal antibody against annexin A6 (Anx A6: red) and a polyclonal one against dysferlin (Dysf:green). The nuclei are labeled with Hoechst stain (blue). A-F: Skeletal muscle biopsy of an individual who has never undergone statin treatment. The boxed areas in A-C are enlarged in D-F. Annexin A6 demarcates the T-tubular system (red), whereas dysferlin outlines the sarcolemma and the nuclear membranes of both muscle and/or satellite cells. G-L: In the skeletal muscle fibers of a myopathic, statin-treated patient, an intracellular accumulation of dysferlin is apparent (H and $\mathbf{I})$; this marker does not colocalize with the annexin A6-labeled T-tubular system (J and $\mathbf{L}$ ). The boxed areas in $\mathbf{G}-\mathbf{I}$ are enlarged in $\mathbf{J}-\mathbf{L}$. Scale bars: $50 \mu \mathrm{m}$ (A-C and G-I), $5 \mu \mathrm{m}$ (D-F and J-L).

membranes (Figure 4C), as well as cholesterol-sensors (Figure 4D), were similar to those in the biopsies of individuals who had never undergone statin therapy. Interestingly, 2 genes (ND1, ATP6) encoding proteins that are involved in mitochondrial activity, were down-regulated in patients who had discontinued statins (Figure 4E).

\section{Discussion}

The underlying cause of statin-associated myotoxicity is unknown, although many different mechanisms have been proposed. These include membrane destabilization, ${ }^{14,15}$ a dearth of GTP-binding proteins, mitochodrial dysfunction, ${ }^{16}$ tRNA immaturity, ${ }^{24}$ interference with the ubiquitine-proteasome pathway, ${ }^{12}$ and the up-regulation of a skeletal muscle atrophy gene. ${ }^{25,26}$

The T-tubular system represents a site of structural weakness in the skeletal muscle of patients who are undergoing statin therapy. ${ }^{13,22}$ Consistent with their role in mediating excitation-contraction coupling, the channels of the T-tubular system surround individual myofibrils and link these with the calcium stores of the sarcoplasmic reticulum. ${ }^{27}$ The T-tubular system is more vulnerable to cholesterol depletion than is the sarcolemma or the sarcoplasmic reticulum, and its structural integrity is readily compromised. ${ }^{22}$

We have previously demonstrated that T-tubular injury can occur during statin therapy ${ }^{22}$ and that it can accom- 
A
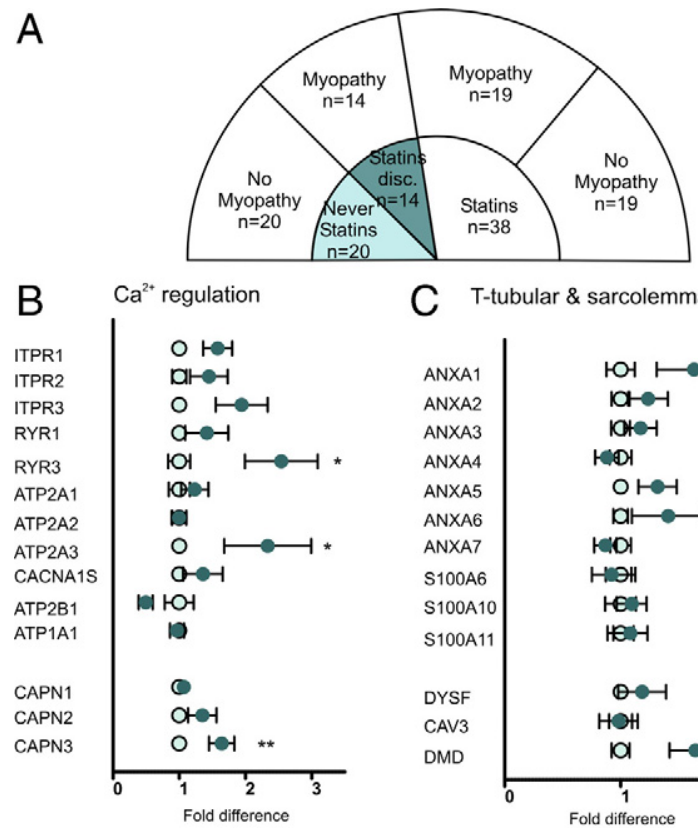

C T-tubular \& sarcolemmal structure

D

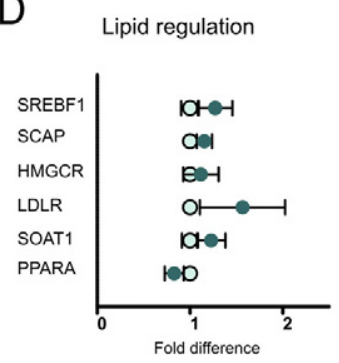

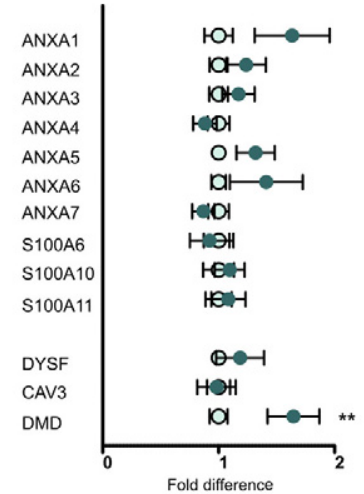

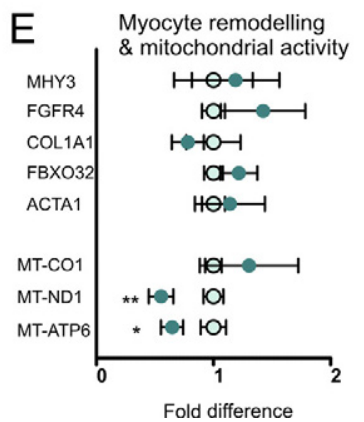

Figure 4. Large-scale normalization of gene expression after discontinuation of statin therapy. A: Color-coded display of the two categories of patients whose biopsies were analyzed for the mRNA levels of genes encoding proteins indicated in $\mathbf{B}-\mathbf{E}$. These patients included the 20 control individuals who had never undergone statin therapy (pale blue) and the 14 myopathic ones who had discontinued their course of treatment with statins (turquoise). B-E: The mRNA levels of 41 candidate genes were determined using a custom TaqMan microarray. These were normalized to the level of 18S RNA. The symbols (color-coded as in A) depict fold differences [reference group: subjects who had never received statin therapy (pale blue)] for each gene product (mean values \pm SEM). B: Several genes that are implicated in $\mathrm{Ca}^{2+}$ homeostasis remain up-regulated: ryanodine receptor 3 (RYR3), the sarco-endoplasmic ATPase 3 (ATP2B3) and calpain 3 (CAPN3). C: Genes that are associated with T-tubular structure and membrane repair are no longer up-regulated, whereas the structural marker of the sarcolemma (dystrophin: DMD) remains highly significantly elevated. D: The gene expression levels of the cholesterol-sensors (SCAP, HMGCR) and the LDL recptor (LDLR) have returned to control values. E: The genes for NADH 1 (MT-ND1) and the mitochondrial ATPase 6 (MT-ATP6) are significantly downregulated. For identification of gene products and $P$ values see Table 1.

pany the upregulation of the ryanodine receptor 3 in patients diagnosed with statin-associated myopathy. ${ }^{13}$ Taken together, these changes point to a potential defect in skeletal muscle $\mathrm{Ca}^{2+}$-regulatory mechanisms. However, the vast majority of patients receiving statin treatment does not experience muscle problems. Therefore, our present study largely focuses on gene expression encoding proteins, which localize to the T-tubular region in skeletal muscle of statin patients who tolerate their treatment well. We deliberately targeted genes involved in the regulation of $\mathrm{Ca}^{2+}$-homeostasis and those that are

known to play a role in plasma membrane repair. The comparison of statin naïve subjects with patients who discontinued statin therapy, constitutes an attempt to assess a potential restitution in gene expression after a cessation of therapy.

\section{Statins and $\mathrm{Ca}^{2+}$ Homeostasis}

Since our preliminary histopathological characterization permitted a discrimination between damaged and undamaged skeletal muscle biopsies, we were able to assess the influence of statin therapy on the expression of genes independently of the clinical findings.

Our data reveal ongoing statin therapy to interfere with the expression of genes that are involved in the maintenance of intracellular $\mathrm{Ca}^{2+}$-homeostasis in skeletal muscle. This finding is consistent with the report of abnormal in vitro contracture tests in muscle from statin-treated patients ${ }^{28}$ and with the results of an experimental study using statin-treated rodents, which showed the resting cytosolic levels of calcium to be elevated in myocytes, without an accompanying increase in the sarcolemmal permeability to this cation. ${ }^{29}$

In skeletal muscle, an increase in the level of sarcoplasmic calcium interferes with excitation-contraction coupling, ${ }^{30}$ which might account for the muscular weakness that is a characteristic trait of statin-associated myopathy. Furthermore, prolonged exposure to high calcium concentrations could activate the skeletal muscle calpains. An activation of calpain in response to treatment with simvastatin has been noted in cultivated human skeletal muscle. ${ }^{10}$ For calpain 3 in particular, a $\mathrm{Ca}^{2+}$-dependent cleavage of the molecule is known to be indispensable for its proteolytic activity. ${ }^{31}$

\section{Statins and Membrane Repair}

The up-regulation of dysferlin, as well as of members of the annexin family and their S100 binding partners, indicates that $\mathrm{Ca}^{2+}$-dependent, dysferlin-mediated membrane repair mechanisms are operative. ${ }^{32}$ In addition to its customary sarcolemmal location, an intracellular, presumably vesicular, accumulation of dysferlin has been described in the skeletal muscle of limb-girdle dystrophy patients, ${ }^{33}$ which accords with our own data. Interestingly, numerous members of the annexin family of genes were up-regulated in the biopsies of asymptomatic patients who were undergoing statin therapy. However, to date, only annexin A1 has been implicated in membrane repair mechanisms. ${ }^{34,35}$ It was surprising that not only well-described repair genes such as dysferlin, and genes encoding several annexins, which bind $\mathrm{Ca}^{2+}$-dependently to the structurally vulnerable T-tubular system, ${ }^{21}$ are up-regulated, but also the gene encoding dystrophin. This points to structural damage within the sarcolemma, which was not apparent in our electron micrographs. 


\section{Skeletal Muscle After Discontinuation of Statin Therapy}

The discontinuation of statin therapy led to an overall normalization of the gene expression profiles. Notable exceptions were the genes for the ryanodine receptor 3 , the sarco-endoplasmic ATPase 3, calpain 3 and dystrophin. In our previous study, the expression levels of the ryanodine receptor 3 and the sarco-endoplasmic ATPase 3 genes were elevated in structurally damaged skeletal muscle. ${ }^{13}$ Since all of the individuals in this group of patients had discontinued the course of treatment owing to muscular problems, we cannot exclude the possibility that several or all of these genes were up-regulated already before statin therapy, rather than being so in consequence of the treatment and remaining thus after its cessation.

An elevation in the gene expression levels of the ryanodine receptor 3 and/or the sarco-endoplasmic ATPase 3 in skeletal muscle might denote a group of individuals who are potentially susceptible to myotoxicity with a tendency toward already elevated sarcoplasmic $\mathrm{Ca}^{2+}$-levels. Conceivably, the raised gene expression levels of calpain 3 coincide with a continued high level of enzyme activity in the wake of a persistent calcium leak.

\section{Statins and Mitochondrial Gene Expression}

Several studies have reported mitochondrial impairment and postulated that mitochondrial dysfunction may underlie statin-associated myotoxicity (rev. by ${ }^{26,36}$ ). Since in our study, several genes encoding proteins implicated in mitochondrial activity were not affected by statin therapy, we are unable to confirm this observation. However, the number of mitochondrial genes we investigated was small; besides, previous findings relating to molecular regulation of mitochondrial activity in cultured cells $\mathrm{s}^{37,38}$ or animal models ${ }^{39}$ may not be readily translatable to human skeletal muscle in vivo.

In our electron micrographs the skeletal muscle mitochondria did not show signs of structural alteration, however, we found 2 genes implicated in mitochondrial activity to be down-regulated in the muscle of patients who had discontinued treatment, which might indicate a pathological reaction.

A recent report showed the upregulation of a gene implicated in skeletal muscle atrophy (FBXO32) in patients with statin-associated myopathy. ${ }^{25}$ However, their human patient cohort was perhaps too small to yield statistically reliable data, since we were unable confirm this result.

An in vitro study addressing the effects of statin treatment on the expression of a broad spectrum of genes, identified 4 genes, all related to cholesterol metabolism to be up-regulated, including HMGCoA reductase, which accords with our own results. ${ }^{9}$ A study by Laaksonen et $\mathrm{al}^{40}$ used whole genome expression profiling of skeletal muscle of 12 statin-treated individuals and 6 placebo controls before and after an 8-week treatment. Their study concluded that high-dose simvastatin correlated with the muscle expression of arachidonate 5-lipoxygen- ase-activating protein. However, this study did not include material from myopathic patients.

\section{Limitations}

An important caveat in the interpretation of our results needs to be mentioned. Due to the limited quantities of available biopsy material, we were unable to ascertain whether the observed changes in gene expression corresponded with changes in protein synthesis. Although the changes in gene expression were highly significant, their magnitude was modest (one- to threefold).

Notwithstanding these reservations, our data point to a fundamental deficiency in the regulation of $\mathrm{Ca}^{2+}$-homeostasis within the skeletal muscle of patients who are undergoing statin therapy. This circumstance should be considered in the development of a new generation of statins whose administration would not be associated with myotoxicity.

\section{Acknowledgments}

We thank Prof R.H. Karas (Boston) for valuable discussion, and Dr. Sarah Potez (Bern) for critically reading the manuscript. The excellent technical assistance of Catherine Allemann, Andrea Hostettler, and Barbara Krieger is gratefully acknowledged.

\section{References}

1. Harper CR, Jacobson TA: The broad spectrum of statin myopathy: from myalgia to rhabdomyolysis. Curr Opin Lipidol 2007, 18:401-408

2. Sathasivam S, Lecky B: Statin induced myopathy. BMJ 2008, 337: a2286. doi: 10.1136/bmj.a2286.:a2286

3. Thompson PD, Clarkson P, Karas RH: Statin-associated myopathy JAMA 2003, 289:1681-1690

4. Bruckert E, Hayem G, Dejager S, Yau C, Begaud B: Mild to moderate muscular symptoms with high-dosage statin therapy in hyperlipidemic patients -the PRIMO Study. Cardiovasc Drugs Ther 2005 , 19:403-414

5. Sinzinger $\mathrm{H}$, Wolfram R, Peskar BA: Muscular side effects of statins J Cardiovasc Pharmacol 2002, 40:163-171

6. Ansell BJ, Fonarow GC, Maki KC, Dicklin MR, Bell M, Davidson MH: Reduced treatment success in lipid management among women with coronary heart disease or risk equivalents: results of a national survey. Am Heart J 2006, 152:976-981

7. Link E, Parish S, Armitage J, Bowman L, Heath S, Matsuda F, Gut I, Lathrop M, Collins R: SLCO1B1 variants and statin-induced myopathy-a genomewide study. N Engl J Med 2008, 359:789-799

8. Voora D, Shah SH, Spasojevic I, Ali S, Reed CR, Salisbury BA, Ginsburg GS: The SLCO1B1*5 genetic variant is associated with statin-induced side effects. J Am Coll Cardiol 2009, 54:1609-1616

9. Morikawa S, Murakami T, Yamazaki H, Izumi A, Saito Y, Hamakubo T, Kodama T: Analysis of the global RNA expression profiles of skeletal muscle cells treated with statins. J Atheroscler Thromb 2005, 12: 121-131

10. Sacher J, Weigl L, Werner M, Szegedi C, Hohenegger M: Delineation of myotoxicity induced by 3-hydroxy-3-methylglutaryl CoA reductase inhibitors in human skeletal muscle cells. J Pharmacol Exp Ther 2005, 314:1032-1041

11. Yu JG, Sewright K, Hubal MJ, Liu JX, Schwartz LM, Hoffman EP, Clarkson PM: Investigation of gene expression in $\mathrm{C}(2) \mathrm{C}(12)$ myotubes following simvastatin application and mechanical strain. J Atheroscler Thromb 2009, 16:21-29

12. Urso ML, Clarkson PM, Hittel D, Hoffman EP, Thompson PD: Changes in ubiquitin proteasome pathway gene expression in skeletal muscle 
with exercise and statins. Arterioscler Thromb Vasc Biol 2005, 25:2560-2566

13. Mohaupt MG, Karas RH, Babiychuk EB, Sanchez-Freire V, Monastyrskaya K, lyer L, Hoppeler H, Breil F, Draeger A: Association between statin-associated myopathy and skeletal muscle damage. CMAJ 2009, 181:E11-E18

14. Langer T, Levy RI: Acute muscular syndrome associated with administration of clofibrate. N Engl J Med 1968, 279:856-858

15. Gharavi AG, Diamond JA, Smith DA, Phillips RA: Niacin-induced myopathy. Am J Cardiol 1994, 74:841-842

16. Ghirlanda G, Oradei A, Manto A, Lippa S, Uccioli L, Caputo S, Greco AV, Littarru GP: Evidence of plasma CoQ10-lowering effect by HMGCoA reductase inhibitors: a double-blind, placebo-controlled study. J Clin Pharmacol 1993, 33:226-229

17. Mueller M, Breil FA, Vogt M, Steiner R, Lippuner K, Popp A, Klossner S, Hoppeler H, Dapp C: Different response to eccentric and concentric training in older men and women. Eur J Appl Physiol 2009, 107:145-153

18. Thompson PD, Clarkson PM, Rosenson RS: An assessment of statin safety by muscle experts. Am J Cardiol 2006, 97:69C-76C

19. Bergstrom J: Percutaneous needle biopsy of skeletal muscle in physiological and clinical research. Scand J Clin Lab Invest 1975, 35:609-616

20. Zaugg P, Djonov V, Füchtbauer EM, Draeger A: Sorting of murine vascular smooth muscle cells during wound healing in the chicken chorioallantoic membrane. Exp Cell Res 1999, 253:599-606

21. Draeger A, Monastyrskaya K, Burkhard FC, Wobus AM, Moss SE, Babiychuk EB: Membrane segregation and downregulation of raft markers during sarcolemmal differentiation in skeletal muscle cells. Dev Biol 2003, 262:324-334

22. Draeger A, Monastyrskaya K, Mohaupt M, Hoppeler H, Savolainen H, Allemann C, Allemann C, Babiychuk E: Statin therapy induces ultrastructural damage in skeletal muscle in patients without myalgia. J Pathol 2006, 210:94-102

23. Glover L, Brown RH Jr: Dysferlin in membrane trafficking and patch repair. Traffic 2007, 8:785-794

24. Moosmann B, Behl C: Selenoprotein synthesis and side-effects of statins. The Lancet 2004, 363:892-894

25. Hanai J, Cao P, Tanksale P, Imamura S, Koshimizu E, Zhao J, Kishi S, Yamashita M, Phillips PS, Sukhatme VP, Lecker SH: The musclespecific ubiquitin ligase atrogin-1/MAFbx mediates statin-induced muscle toxicity. J Clin Invest 2007, 117:3940-3951

26. Mallinson JE, Constantin-Teodosiu D, Sidaway J, Westwood FR, Greenhaff PL: Blunted Akt/FOXO signalling and activation of genes controlling atrophy and fuel use in statin myopathy. J Physiol 2009, 587:219-230

27. Franzini-Armstrong C: Architecture and regulation of the $\mathrm{Ca} 2+$ deliv- ery system in muscle cells. Appl Physiol Nutr Metab 2009, 34:323-327

28. Guis S, Figarella-Branger D, Mattei JP, Nicoli F, Le Fur Y, KozakRibbens G, Pellissier JF, Cozzone PJ, Amabile N, Bendahan D: In vivo and in vitro characterization of skeletal muscle metabolism in patients with statin-induced adverse effects. Arthritis Rheum 2006, 55:551-557

29. Liantonio A, Giannuzzi V, Cippone V, Camerino GM, Pierno S, Camerino DC: Fluvastatin and atorvastatin affect calcium homeostasis of rat skeletal muscle fibers in vivo and in vitro by impairing the sarcoplasmic reticulum/mitochondria Ca2+-release system. J Pharmacol Exp Ther 2007, 321:626-634

30. Lamb GD, Junankar PR, Stephenson DG: Raised intracellular [Ca2+] abolishes excitation-contraction coupling in skeletal muscle fibres of rat and toad. J Physiol 1995, 489:349-362

31. Verburg E, Murphy RM, Richard I, Lamb GD: Involvement of calpains in $\mathrm{Ca} 2+$-induced disruption of excitation-contraction coupling in mammalian skeletal muscle fibers. Am J Physiol Cell Physiol 2009, 296:C1115-C1122

32. Han R, Campbell KP: Dysferlin and muscle membrane repair. Curr Opin Cell Biol 2007, 19:409-416

33. Piccolo F, Moore SA, Ford GC, Campbell KP: Intracellular accumulation and reduced sarcolemmal expression of dysferlin in limbgirdle muscular dystrophies. Ann Neurol 2000, 48:902-912

34. McNeil AK, Rescher U, Gerke V, McNeil PL: Requirement for annexin A1 in plasma membrane repair. J Biol Chem 2006, 281:35202-35207

35. Babiychuk EB, Monastyrskaya K, Potez S, Draeger A: Intracellular $\mathrm{Ca}(2+)$ operates a switch between repair and lysis of streptolysin O-perforated cells. Cell Death Differ 2009, 16:1126-1134

36. Golomb BA, Evans MA: Statin adverse effects : a review of the literature and evidence for a mitochondrial mechanism. Am J Cardiovasc Drugs 2008, 8:373-418

37. van Vliet AK, Negre-Aminou P, van Thiel GC, Bolhuis PA, Cohen LH Action of lovastatin, simvastatin, and pravastatin on sterol synthesis and their antiproliferative effect in cultured myoblasts from human striated muscle. Biochem Pharmacol 1996, 52:1387-1392

38. Kaufmann P, Torok M, Zahno A, Waldhauser KM, Brecht K, Krahenbuhl S: Toxicity of statins on rat skeletal muscle mitochondria. Cell Mol Life Sci 2006, 63:2415-2325

39. Westwood FR, Bigley A, Randall K, Marsden AM, Scott RC: Statininduced muscle necrosis in the rat: distribution, development, and fibre selectivity. Toxicol Pathol 2005, 33:246-257

40. Laaksonen R, Katajamaa M, Paiva H, Sysi-Aho M, Saarinen L, Junni P, Lutjohann D, Smet J, Van Coster R, Seppanen-Laakso T, Lehtimaki T, Soini J, Oresic M: A systems biology strategy reveals biological pathways and plasma biomarker candidates for potentially toxic statin-induced changes in muscle. PLoS ONE 2006, \%20,1:e97 